Review

\title{
Cosmology with the Nearby Supernova Factory
}

\author{
M. Kerschhaggl ${ }^{\mathrm{a}, *}$, G. Aldering ${ }^{\mathrm{b}}$, P. Antilogus ${ }^{\mathrm{c}}$, C. Aragon $^{\mathrm{b}}$, S. Bailey ${ }^{\mathrm{b}}$, C. Baltay ${ }^{\mathrm{d}}$, S. Bongard ${ }^{\mathrm{c}}$, \\ C. Buton ${ }^{\mathrm{a}}$, A. Canto ${ }^{\mathrm{c}}$, M. Childress ${ }^{\mathrm{b}, \mathrm{e}}, \mathrm{N}$, Chotard ${ }^{\mathrm{f}}$, Y. Copin ${ }^{\mathrm{f}}$, H.K. Fakhouri b,e \\ E. Gangler ${ }^{\mathrm{f}}$, E.Y. Hsiao ${ }^{\mathrm{b}}$, M. Kowalski ${ }^{\mathrm{a}}$, S. Loken ${ }^{\mathrm{b}}$, P. Nugent ${ }^{\mathrm{b}, \mathrm{g}}$, K. Paech $^{\mathrm{a}}$, R. Pain ${ }^{\mathrm{c}}$, \\ E. Pecontal ${ }^{\text {h }}$, R. Pereira ${ }^{f}$, S. Perlmutter ${ }^{\text {bee }}$, D. Rabinowitz ${ }^{d}$, K. Runge $^{b}$, R. Scalzo $^{d}$, \\ G. Smadja ${ }^{f}$, C. Tao ${ }^{\mathrm{i}, \mathrm{j}}$, R.C. Thomas ${ }^{\mathrm{b}, \mathrm{g}}, \mathrm{C}$. Wu ${ }^{\mathrm{c}}$ \\ a Physikalisches Institut Universitat Bonn, Nussallee 1253115 Bonn, Germany \\ b Physics Division, Lawrence Berkeley National Laboratory, 1 Cyclotron Road, Berkeley, CA 94720, United States \\ ${ }^{\mathrm{c}}$ Laboratoire de Physique Nucléaire et des Hautes Énergies, Université Pierre et Marie Curie Paris 6, Université Paris, Diderot Paris 7, CNRS-IN2P3, 4 place Jussieu, \\ 75252 Paris Cedex 05, France \\ d Department of Physics, Yale University, New Haven, CT 06250-8121, United States \\ e Department of Physics, University of California Berkeley, Berkeley, CA 94720, United States \\ ${ }^{\mathrm{f}}$ Université de Lyon, 69622, Université de Lyon 1, CNRS/IN2P3, Institut de Physique Nucléaire de Lyon, France \\ g Computational Cosmology Center, Computational Research Division, Lawrence Berkeley National Laboratory, 1 Cyclotron Road MS 50B-4206, Berkeley, \\ CA 94611, United States \\ ${ }^{\mathrm{h}}$ Centre de Recherche Astronomique de Lyon, Université Lyon 1, 9 Avenue Charles André, 69561 Saint Genis Laval Cedex, France \\ ${ }^{\mathrm{i}}$ CPPM, 163 Av. Luminy, 13288 Marseille Cedex 09, France \\ j THCA, Tsinghua U., Beijing, China
}

\section{A R T I C L E I N F O}

\section{Keywords:}

Supernovae

General-cosmology

Observations

\begin{abstract}
A B S T R A C T
The Nearby Supernova Factory (SNfactory) is currently finishing its first survey of low redshift $(0.03<z<0.08)$ type la supernovae. These data add to the understanding of the expansion history of the universe. Observations are performed using the Supernova Integral Field Spectrograph (SNIFS), an integral field spectrograph delivering full spectrophotometric information of the target. The corresponding dataset aims at the inference of the Hubble diagram zero point with unprecedented accuracy. Moreover, the data offers a variety of related physical studies such as the understanding of progenitor systems, explosion scenarios and host galaxy characteristics. The SNfactory is also working on novel methods accessible with spectro-photometric measurements, reducing systematic uncertainties and improving the statistical power of the SN data.
\end{abstract}

(C) 2011 Elsevier B.V. All rights reserved.

\section{Introduction}

Type Ia Supernovae (SN Ia) are used as standard candles in observational cosmology since their empirical standardizability makes them ideal probes for testing the expansion history of the universe. About a decade ago the observations of high redshift SN Ia led to the conclusion that the expansion of the universe is accelerating [1,2]. The magnitudes of high- $z$ SN Ia are dimmer than predicted by a universe filled with matter only. One possible interpretation for those high- $z$ SNe is that they must have receded further away due to recent acceleration in the cosmic expansion. The introduction of Dark Energy to the Einstein equations in the form of a cosmological constant $\Lambda$ can explain the observational results since it leads to a

\footnotetext{
* Corresponding author.

E-mail address: mkersch@physik.uni-bonn.de (M. Kerschhaggl).
} 

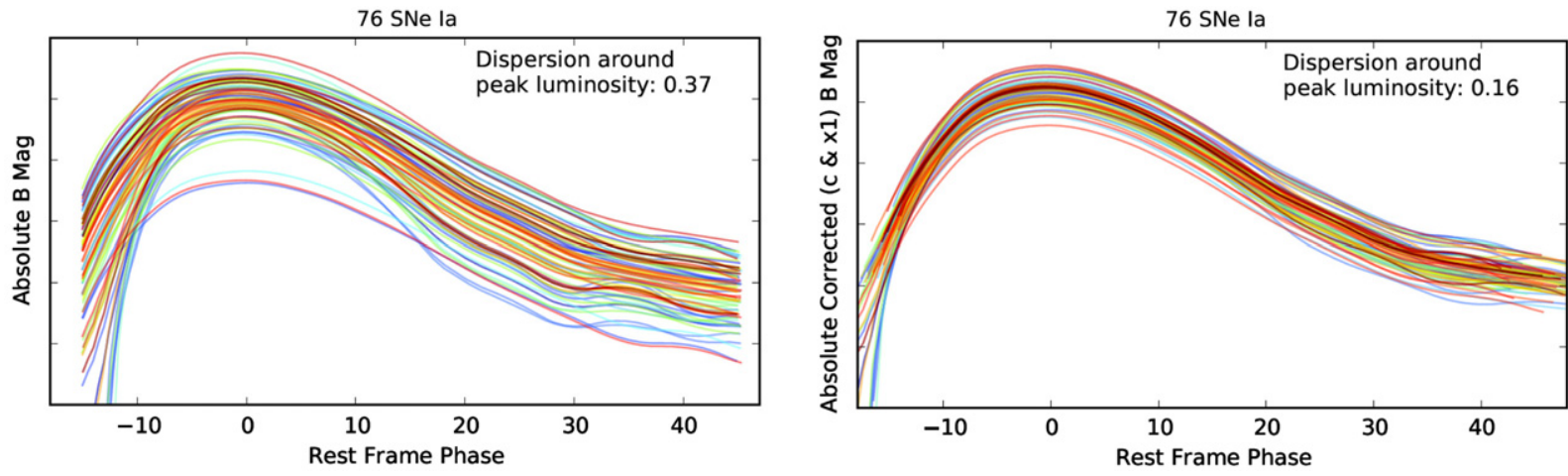

Fig. 1. Lightcurves of $76 \mathrm{SNe}$ la from the Nearby Supernova Factory dataset. (left) Before and (right) after empirical standardization.
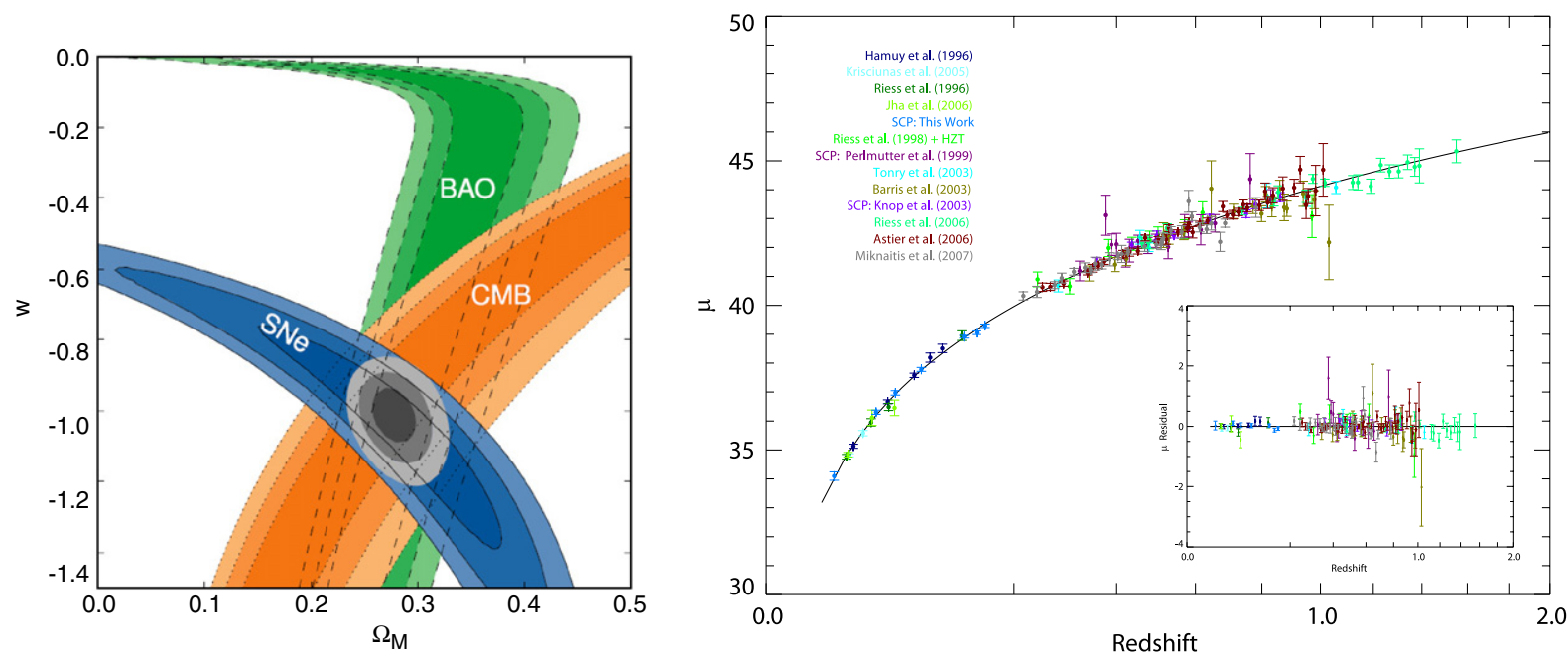

Fig. 2. (left) $1 \sigma, 2 \sigma$ and $3 \sigma$ constraints in $\Omega_{M}-\omega$-space of SN Ia data combined with data from BAO and CMB (without systematics) [4]. (right) Hubble diagram of combined SNe la datasets [5].

negative pressure in the equation of state $p=\omega \rho$, counteracting the gravitational attractive force. The currently accepted concordance model of cosmology, the so-called $\Lambda \mathrm{CDM}$ model, thus features Dark Energy as an additional contributor to the overall energy density, $\Omega$, in the universe. Apart from Dark Matter and the commonly known baryonic matter, Dark Energy is by far the biggest component in this model, making up $\sim 3 / 4$ of the energy content.

\section{SN Ia cosmology}

SN Ia are driven by the thermonuclear explosion of a white dwarf within a binary system comprising a massive donor star. In the scenario of such a single degenerate model the white dwarf accretes matter from its companion until approaching the Chandrasekhar mass limit $M_{\mathrm{Ch}}$ of $\sim 1.4$ solar masses. Once this limit is reached, gravitational attraction overcomes the electron degeneracy pressure. However, before such a scenario would lead to a collapse, the core of the white dwarf reaches temperatures triggering the ignition of carbon fusion terminating in a supernova explosion. Even if the physical parameters, such as the supernova progenitor mass, appear to be the same for most of the SN la they are not standard candles by themselves. Fig. 1(left) shows the intrinsic dispersion of $\sim 0.37$ mag observed in a set of SN Ia lightcurves. However, as pointed out in [3] SN la that have bluer colors or broader lightcurves appear to be intrinsically brighter. This empirical handle, the so-called Phillips relation, on the supernova intrinsic luminosity allows SN Ia to be standardizable. This reduces the luminosity dispersion in SNe Ia down to $\sim 0.16$ mag (see Fig. 1(right)). Thus standardized, SNe Ia are the established standard candles of cosmology adding to the understanding of the universe and complementing other sources such as data from the cosmic microwave background (CMB) and baryonic acoustic oscillations (BAO). The combination of these independent data yields accurate constraints on the $\Lambda \mathrm{CDM}$ cosmological parameters such as Dark Energy density $\Omega_{\Lambda}$, dark matter density $\Omega_{M}$ and equation of state parameter $\omega$. Fig. 2(left) illustrates the impact of SN Ia data on the parameter fits which are consistent with the existence of a cosmological constant, i.e. $\omega=-0.997_{-0.082}^{+0.077}$ [4]. The right plot in Fig. 2 shows the Hubble diagram (HD) of various SNe Ia data combined in the Union compilation [5]. While high- $z$ SNe deliver information 
about the cosmic expansion w.r.t. the nearby SNe and thus allow the inference of $\Omega_{\Lambda}$ and $\Omega_{M}$, nearby SNe are crucial for the correct normalization of the HD since they constrain the $\mathrm{SN}$ absolute luminosity.

\section{The Nearby Supernova Factory}

The Nearby Supernova Factory (SNfactory) is a project designed for the observation of SNe Ia within the nearby Hubble flow, i.e. the redshift range of $0.03<z<0.08$ [6]. In this regime the SNe in the HD are neither dominated by peculiar motions nor does cosmic acceleration have a significant impact, i.e. the linear Hubble law $H_{0} d \propto z$ applies to good approximation.

The science goal of the SNfactory is the collection of a high quality dataset comprising full spectro-photometric information of low- $z$ SNe Ia. These data can be used to determine the HD normalization with unprecedented accuracy as well as for studying SN Ia physics including progenitors, host galaxies, dust extinction and explosion mechanisms.

The SNfactory observation and data processing chain operated as follows: Images from the asteroid searching NEAT project and the Palomar-QUEST Consortium were transferred from Palomar to the Lawrence Berkeley National Laboratory via a custom-built high-speed wireless network and processed in order to discover SN candidates. SN candidates were spectroscopically screened and if identified as type Ia followed every 2-3 nights using the Supernova Integral Field Spectrograph (SNIFS) mounted at the UH88-inch telescope located at Mauna Kea Hawaii.

\subsection{SNIFS}

SNIFS is a two-channel integral field spectrograph covering a wavelength range of 320-550 nm (blue) and 510-1100 $\mathrm{nm}$ (red) with a sampling of $0.238 \mathrm{~nm} /$ pixel and $0.293 \mathrm{~nm} /$ pixel, respectively [7]. Light from an object observed with SNIFS passes through a microlens array integral field unit $\left(15 \times 15\right.$ lenses) before entering each channel covering a $6^{\prime \prime} \times 6^{\prime \prime}$ field of view (FOV) at a spatial sampling of $0.43^{\prime \prime}$. Once dispersed in each spectrographic channel, featuring $2048 \times 4096$ pixel CCD detectors, this gives 225 distinct spectra of the observed target. These data are stored in so-called cubes, containing the full $3 \mathrm{D}$ information of the source, i.e. photon rate as a function of image coordinates $x, y$ and wavelength $\lambda$. Observing a target over time thus allows the full spectro-photometric evolution of a SN to be inferred. Each data cube contains contributions from the SN itself, its host galaxy and the sky level. The data are analyzed using a semi-analytical model for the point spread function (PSF) of the instrument retrieving spectra for all contributing sources: the SN, its host galaxy, and the sky. Photometric conditions for each observation are inferred from a photometric (P-)channel detecting light from defined standard stars propagating through a defined set of filters. A more thorough discussion of the spectrographic pipeline is given in [8].

\subsection{Spectro-photometry}

Once an SN has been followed with SNIFS over time a spectral time series of the source can be compiled. Typically the SNfactory performs 15 observations per target starting at or before maximum brightness. Fig. 3 shows the spectral evolution of a SN. According to this figure the photometric data necessary for constructing an SN lightcurve can be inferred from the spectral data by integrating over any given filter set. As such, the SNfactory data is not confined to the bandwidth of some instrument specific filter, unlike photometric SN Ia surveys, but encodes the full spectral and evolutionary information of the source plus its host galaxy within the given spectral and spatial resolution of SNIFS.

At the moment the typical level of systematics of photometric measurements propagating to the cosmological observables is in the \% regime. In order to optimally make use of the available statistics it is thus essential to reach at a better systematics control of the data aiming for a $<1 \%$ precision. This goal can be achieved by employing improved statistical methods and modeling to the full spectral information of an SN. For instance, the high accuracy spectro-photometric calibration methodology applied to SNIFS spectra directly impacts the quality of the supernova absolute magnitude measurement. Spectra from standard stars observed in between SN observations are used to disentangle the atmospheric extinction physical components. A full spectral atmospheric correction computed each observation night is then applied to all SN spectra which allows generation of synthetic photometry with high precision [9].

\section{SNfactory results}

The SNfactory has now completed its first phase of data taking and is close to a first data release. However, a number of scientific results based on this unique dataset have been already published (see e.g. [8,10-13]).

The SNfactory has up to now gathered around 200 SNe la with full spectral data.

\subsection{Spectral flux ratio standardization}

As described above the SNfactory dataset is unique for its full spectro-temporal information. It can be used to improve the systematic limitations of the HD by studying spectral features of SNe spectra that correlate with brightness. Such features 


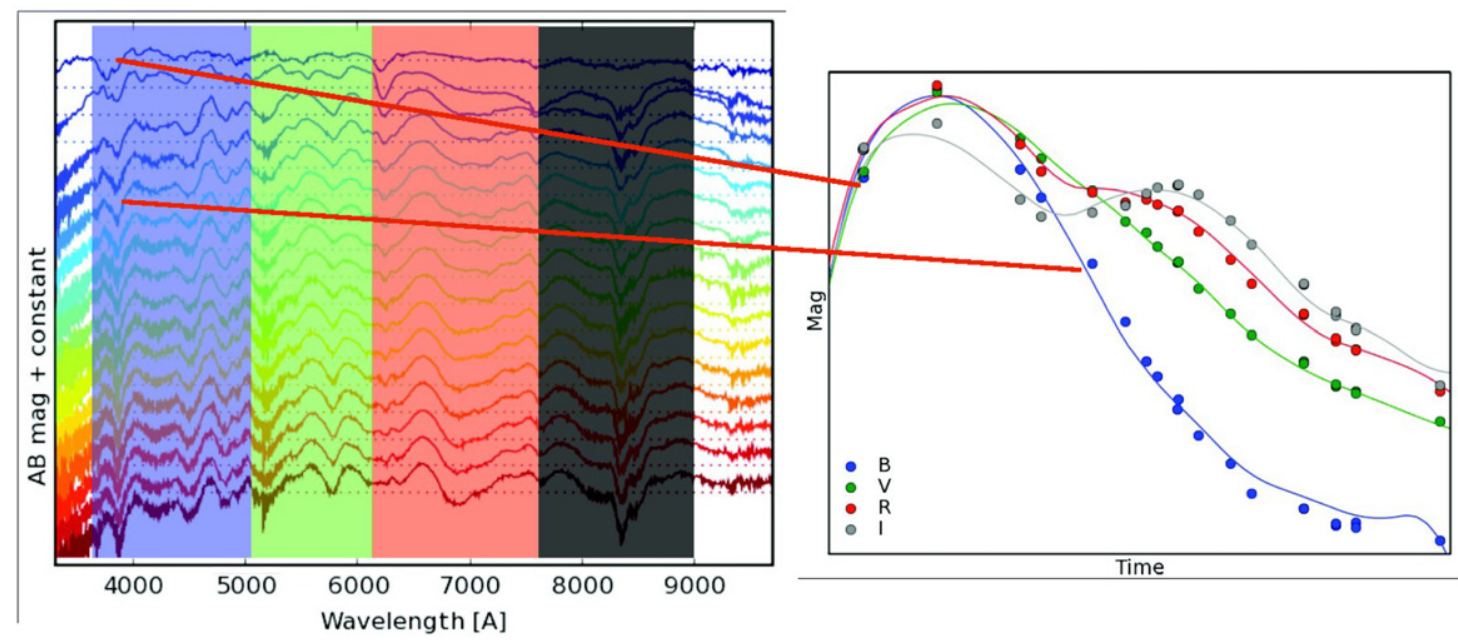

Fig. 3. (left) SN spectral evolution depicted in color code from blue (early) to red (late). (right) Lightcurve data as synthesized from spectra using a filter set as indicated by the colored shaded regions in the left plot. (For interpretation of the references to colour in this figure legend, the reader is referred to the web version of this article.)

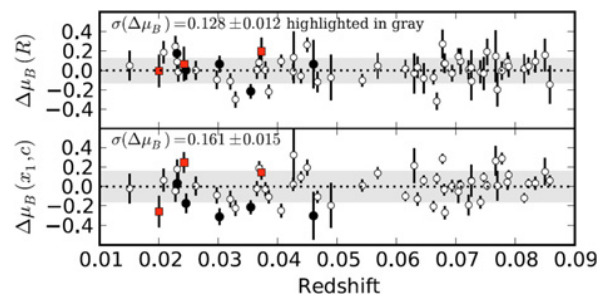

Fig. 4. (top) HD residuals employing the flux ratio one-parameter standardization explained in the text [11]. (bottom) The same data using the SALT2 two-parameter lightcurve fitter [14].

are for instance the ratios between different absorption lines in the spectrum, flux ratios at different wavelengths, feature velocities (i.e. blue shifted lines from the exploding ejecta material) or pseudo-equivalent widths of absorption features corresponding to the abundance of absorbing material. In [11] all possible flux ratios $R_{x} y=F_{x} / F_{y}$ for all wavelength combinations $(x, y)$ in the SN spectra within \pm 2.5 restframe days of B-band maximum light were correlated to the residuals in the HD. The flux ratio with highest impact on the HD dispersion was identified. This was done using a training sample of SN Ia and validated for an independent sample yielding the flux ratio $R_{642 / 433}$ as being maximally correlated with the Hubble residual with a Pearson coefficient of $\rho=0.96$. Correcting the magnitudes of SNe in the HD employing $R_{642 / 433}$ is shown in Fig. 4. This shows that the dispersion in the HD was reduced from 0.161 mag to 0.128 mag compared to the standard approach. This increases the weight of each SN by $60 \%$.

\subsection{SN Ia physics: SN 2007 if}

Apart from reducing systematics inherent to modern cosmological measurements, the SNfactory data is also suitable for the study of SN Ia physics. The spectra at hand allow for testing explosion models, dust modeling and inference of progenitor masses and element abundances. Although the single degenerate model of type Ia physics appears to cover most SNe there are some cases of spectroscopically classified SN Ia defying the predictions of the model. Beside the standard SN Ia there are cases known with progenitor masses being significantly under or exceeding the canonical Chandrasekhar mass. In the double degenerate model two white dwarfs in a binary system are merged to form a SN Ia exceeding $M_{\mathrm{Ch}}$. One example of such a Super Chandrasekhar SN Ia observed with SNIFS is SN 2007if [10]. The SNIFS data of this object were fitted with progenitor mass models yielding a total mass untypical for the otherwise spectroscopically clear case of a type Ia. Fig. 5 shows a fit to the bolometric lightcurve of SN 2007if compatible with a Ni mass of 1.72 solar masses. So the amount of Ni in SN 2007if itself exceeds the limit of $M_{\mathrm{Ch}}=\sim 1.4$ solar masses.

\section{Conclusion}

The SNfactory has compiled a dataset of 200 nearby spectro-photometric SN Ia observed during the last few years. This invaluable spectral dataset is used to increase statistical power and control of systematics necessary for modern precision 


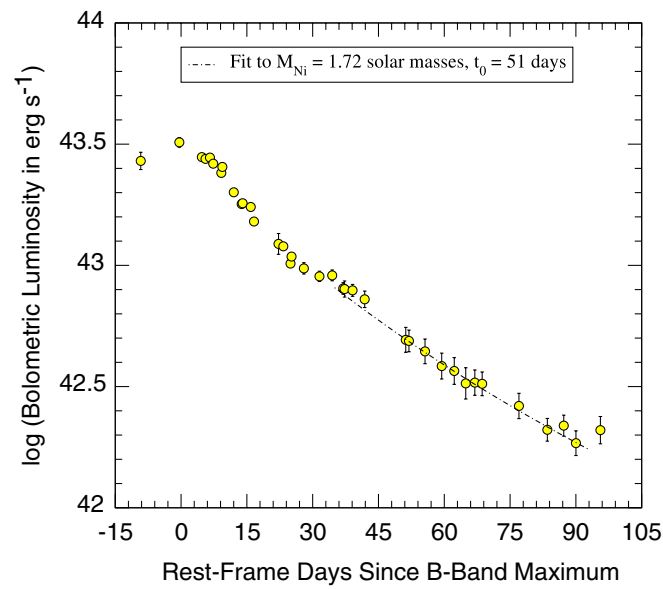

Fig. 5. Bolometric lightcurve of SN 2007if as measured with SNIFS [10].

cosmology. It will allow for an unprecedented accuracy in the determination of the HD zero point. New and more robust standardization methods, such as correlations to flux ratios, to reduce the intrinsic dispersion of SN Ia brightness are applied using the full spectral information of the data. The same data provide unique input for probing, modeling and understanding SN Ia physics.

Currently, the SNfactory is engaged in preparation for a second phase of the project with improved techniques and methods.

\section{References}

[1] A.G. Riess, et al., Astronomical Journal 116 (1998) 1009

[2] S. Perlmutter, et al., Astrophysical Journal 517 (1999) 565.

[3] M.M. Phillips, Astrophysical Journal 413 (1993) L105-L108.

[4] R. Amanullah, et al., Astrophysical Journal 716 (2010) 712-738.

[5] M. Kowalski, et al., Astrophysical Journal 686 (2008) 749-778.

[6] G. Aldering, et al., Society of Photo-Optical Instrumentation Engineers (SPIE) Conference Series, vol. 4836, 2002, pp. 61-72.

[7] B. Lantz, et al., Society of Photo-Optical Instrumentation Engineers (SPIE) Conference Series, vol. 5249, 2004, pp. 146-155.

[8] G. Aldering, et al., Astrophysical Journal 650 (2006) 510-527.

[9] C. Buton, et al., SF2A-2007, in: Proceedings of the Annual Meeting of the French Society of Astronomy and Astrophysics, 2007, pp. $288-292$.

[10] R.A. Scalzo, et al., Astrophysical Journal 713 (2010) 1073-1094.

[11] S. Bailey, et al., Astronomy and Astrophysics 500 (2009) L17-L20.

[12] C. Aspin, et al., Astrophysical Journal 692 (2009) L67-L71.

[13] R.C. Thomas, et al., Astrophysical Journal 654 (2007) L53-L56.

[14] J. Guy, et al., Astronomy and Astrophysics 466 (2007) 11-21. 